\title{
Full-Course Psychological Intervention for Puerperas with COVID-19
}

\author{
Xiao Y, Li W and Zhang $X^{*}$ \\ Department and Institute of Infectious Disease, Huazhong University of Science and Technology, \\ China
}

*Corresponding author: Xiaoyun Zhang, Department and Institute of Infectious Disease, Tongji Hospital, Tongji Medical College, Huazhong University of Science and Technology, No. 1095, Jiefang Avenue, Wuhan 430030, China, Tel: 86.27.8366.5010; Email: tongjizxy@aliyun.com

\section{Research Article}

Volume 4 Special Issue 1

Received Date: May 22, 2020

Published Date: June 29, 2020

DOI: $10.23880 /$ nhij-16000S1-002

\section{Abstract}

Purpose: An outbreak of Coronavirus Disease 2019 (COVID-19) caused by severe acute respiratory syndrome coronavirus 2 (SARS-CoV-2) emerged in Wuhan since December 2019. Anxiety and depression tendencies are common in puerperas with COVID-19. We aimed to delineate and compare the psychological status of puerperas infected with COVID-19.

Method: In this descriptive study, a postpartum psychological care team has been formed to supply full-course psychological intervention, and a dynamic changement of psychological status of 9 patients with COVID-19 was analyzed.

Results: psychological status evaluation results showed that anxiety and depression scores reach a peak during 3-10 days after cesarean section, and then gradually declined. 88.89\% (8/9) and 11.11\% (1 / 9) of puerperas demonstrated mild and moderate anxiety experience respectively. 33.33\% (3/9) of puerperas showed a tendency to depression. The fear scores of 88.89\% (8/9) puerperas were $\geq 5$ points, the peak value occurred at the first 24 hours post-operative, and gradually declined.

Conclusion: The SARS-CoV-2 infection may affect psychological status of puerperas, individual psychological intervention could decrease the degree of anxiety, depress and fear.

Keywords: New coronavirus; Puerpera; Psychological care

\section{Introduction}

Since December 2019, a new coronavirus, officially designated as the severe acute respiratory syndrome coronavirus 2 (SARS-CoV-2), is a member of Beta-CoV lineage $\mathrm{B}$, which was first identified in Wuhan by the Chinese Center for Disease Control and Prevention (CDC) [1]. As of May 2020, the number of SARS-CoV-2 cases globally had eclipsed 4.9 million cases all over the world, largely exceeding the total number of SARS cases during the 2003 epidemic, and more than 320, 000 people had now died. The puerperium is a special period marked by women intense vulnerability. Pain and discomfort caused by parturition and the sudden changement of the puerpera's self-role make it easy to evoke various psychologic problems. Depression and anxiety were the most common emotional problems for mothers [2]. Pregnant women with COVID-19 pneumonia need to be quarantined in isolation ward immediately after delivery, and mother and newborn were quarantined in different departments. It makes depression and anxiety and other negative emotions are more likely to happen. In our study, we performed a full-course adequate psychological care to puerperas to alleviate their negative emotions and analyzed psychologic status of Puerperas with COVID-19 in the postoperative period of cesarean section. These findings may help us extend our understanding of individual nursing care for special patients in the SARS-CoV-2 infection. 


\section{Study Participants and Data Collection}

From 13 January to February 14th 2020, 9 puerperas with pneumonia were admitted to the isolation ward at Wuhan Tongji hospital. all of them were identified as laboratoryconfirmed SARS-CoV-2 infection, and were directly sent to the quarantine ward for isolation treatment after cesarean section, all patients demonstrated percutaneous oxygen saturation (Sp02) $\geq 96 \%$ with nasal cannula oxygen (3L/ min) which not reaching criteria of severe COVID-19 were considered as mild according to the Guidelines for diagnosis and management of COVID-19 (6th edition, in Chinese) issued by the National Health Commission of China. Table 1 showed the clinic characteristics of these 9 puerperas.

\begin{tabular}{|c|c|c|c|c|c|c|c|c|c|}
\hline \multirow[b]{2}{*}{ Number } & \multirow[b]{2}{*}{1} & \multicolumn{7}{|c|}{ The clinic characteristics of puerperas } & \multirow[b]{2}{*}{9} \\
\hline & & 2 & 3 & 4 & 5 & 6 & 7 & 8 & \\
\hline Age & 31 & 34 & 34 & 33 & 30 & 31 & 30 & 30 & 30 \\
\hline $\begin{array}{l}\text { Number of } \\
\text { deliveries }\end{array}$ & 2 & 2 & 1 & 2 & 2 & 2 & 1 & 1 & 1 \\
\hline $\begin{array}{c}\text { Gestational week } \\
\text { at birth }\end{array}$ & $41+2$ & $38+2$ & 40 & 37 & 40 & $36+4$ & $38+4$ & $39+1$ & $36+5$ \\
\hline Past history & None & None & Hypothyroidism & None & None & $\begin{array}{l}\text { Fatty } \\
\text { liver }\end{array}$ & $\begin{array}{l}\text { Polycystic } \\
\text { Ovary } \\
\text { Syndrome } \\
\end{array}$ & None & HBV \\
\hline $\begin{array}{l}\text { Nucleic acid test } \\
\text { results }\end{array}$ & + & + & + & + & + & + & + & + & + \\
\hline $\begin{array}{c}\text { Oxygen } \\
\text { saturation during } \\
\text { hospitalization }\end{array}$ & $\geq 96 \%$ & $\geq 96 \%$ & $\geq 96 \%$ & $\geq 96 \%$ & $\geq 96 \%$ & $\geq 96 \%$ & $\geq 96 \%$ & $\geq 96 \%$ & $\geq 96 \%$ \\
\hline $\begin{array}{l}\text { Family members } \\
\text { with Covid-19 }\end{array}$ & husband & None & Newborn & None & None & None & None & None & None \\
\hline $\begin{array}{l}\text { Nesborn virus } \\
\text { nucleic acid test } \\
\text { results }\end{array}$ & - & + & - & - & - & - & - & - & - \\
\hline $\begin{array}{l}\text { Fever days after } \\
\text { hospitalization }\end{array}$ & 7 & 4 & 2 & 0 & 3 & 0 & 0 & 0 & 0 \\
\hline Cough degree & Mild & Mild & Mild & Severe & Mild & None & Mild & Mild & Mild \\
\hline Gasp degree & None & None & None & Mild & Mild & None & None & None & None \\
\hline
\end{tabular}

Table 1: The clinic characteristics of these 9 puerperas.

\section{Mental State Assessment Tools}

\section{Anxiety Assessment}

The Generalized Anxiety Disorder (GAD-7) was used to evaluate the degree of anxiety. GAD-7 was proposed by Spitzer RL, etc. $[3,4]$, contains seven entries, with a total score of 21 points, $0 \sim 4$ points means no anxiety; 5-9 points may be mild anxiety, it is suggested to consult a psychiatrist or psychologist Workers; 10-13 may represent moderate anxiety, $\geq 14$ points means moderate and severe anxiety.

\section{Depression Assessment}

The Edinburgh postnatal depression scale (EPDS) was used to evaluate the depression status. The EPDS was compiled by Cox in 1978. In 1998, Lee of the Chinese University of Hong Kong was compiled into the Chinese version. Its Cronbach's alpha was 0.89 . The scale has a total of 10 items, each item is assigned a score of 0 to 3 according to the severity of symptoms, and the total score is 30 points. Most scholars recommend a score of $\geq 12$ as the critical value, and those with a score of $\geq 12$ have a tendency to postpartum depression $[5,6]$.

\section{Fear Assessment}

Using a self-made fear score table, 0 points represent no fear, 10 points represent the strongest sense of fear, and women were encouraged to score according to their fear of disease and environment. The higher the score they choose, the 
stronger fear they felt.

Specific implementation of psychological nursing intervention during the hospitalization.

\section{Establishment of Psychological Intervention Group and Assessment of Puerpera Mental State}

Patients with Covid-19 pneumonia may progress rapidly. To reduce the incidence of intrauterine distress, for the safety of mothers and fetus, cesarean section was performed for the full-term pregnant patients. Compared with natural delivery, cesarean section is more likely to evoke bad mood to pregnant woman [7]. We coordinated and selected 6 wellexperienced nurses and one doctor to set up a postpartum psychological care team. During the hospitalization, individual psychological cares were performed, and the psychological status was recorded at different time point.

Within 24 hours, 72 hours, 1 week after surgery, the postpartum psychological care team members used GAD7, EPDS and self-made fear scores to assess patients' status of anxiety, depression and fear face to face. 1 month after surgery, all patients were discharged and were segregated at Community isolation point, no visiting was allowed, the postpartum psychological care team members performed assessment of anxiety, depression and fear with We Chat or telephone follow up. The results of these nine cases showed that anxiety and depression scores reach a peak during 3-10 days after surgery, and gradually declined. $88.89 \%(8 / 9)$ of women showed mild anxiety experience, $11.11 \%(1 / 9)$ with moderate anxiety, and 33.33\% (3/9) of them displayed a tendency to depression. The fear scores of $88.89 \%(8 / 9)$ puerperas were $\geq 5$ points, the peak value occurred at the first 24hours post-operative, and gradually declined. The results were shown in Table 2 .

\begin{tabular}{|c|c|c|c|c|c|c|c|c|c|c|c|c|}
\hline \multicolumn{10}{|c|}{ Puerpera psychological status evaluation results (n = 9) } \\
\hline \multirow{2}{*}{ Number } & \multicolumn{9}{|c|}{ GAD-7score } & \multicolumn{4}{c|}{ EPDSscore } & \multicolumn{5}{c|}{ Fear score } \\
\cline { 2 - 14 } & $\mathbf{2 4 h}$ & $\mathbf{7 2 h - 9 6 h}$ & $\mathbf{8 - 1 0 d}$ & 1Month & $\mathbf{2 4 h}$ & $\mathbf{7 2 h - 9 6 h}$ & $\mathbf{8 - 1 0 d}$ & 1Month & $\mathbf{2 4 h}$ & $\mathbf{7 2 h - 9 6 h}$ & $\mathbf{8 - 1 0 d}$ & 1Month \\
\hline 1 & 7 & 9 & 9 & 7 & 8 & 15 & 16 & 11 & 8 & 6 & 4 & 2 \\
\hline 2 & 8 & 11 & 13 & 9 & 12 & 20 & 18 & 9 & 9 & 8 & 5 & 2 \\
\hline 3 & 6 & 8 & 4 & 6 & 7 & 11 & 11 & 8 & 7 & 5 & 4 & 1 \\
\hline 4 & 3 & 3 & 3 & 4 & 6 & 8 & 8 & 5 & 7 & 4 & 3 & 1 \\
\hline 5 & 5 & 6 & 6 & 4 & 8 & 11 & 9 & 6 & 8 & 5 & 4 & 1 \\
\hline 6 & 5 & 7 & 6 & 3 & 6 & 9 & 10 & 7 & 8 & 4 & 3 & 1 \\
\hline 7 & 5 & 9 & 6 & 4 & 8 & 11 & 10 & 5 & 9 & 6 & 3 & 2 \\
\hline 8 & 4 & 7 & 7 & 4 & 8 & 10 & 9 & 4 & 4 & 4 & 3 & 1 \\
\hline 9 & 4 & 5 & 4 & 4 & 10 & 14 & 13 & 5 & 9 & 7 & 5 & 2 \\
\hline average & 5.22 & 7.22 & 6.44 & 5.00 & 8.11 & 12.11 & 11.56 & 6.67 & 7.67 & 5.44 & 3.78 & 1.44 \\
\hline
\end{tabular}

Table 2: The peak value occured at the first 24 hours post-operative.

\section{Psychological Problems and Nursing Measures within the First 24 Hours after Operation}

Local anesthesia was used in the cesarean section operation; the fear of the strange environment and the medical staffs who have worn personal protective equipment was the most important emotion of the mother at this stage. In addition, the severe pain of surgical wounds and physical discomfort would increase the puerpera's concerns of their own diseases. Therefore, when the patient had completed the operation and been escorted to our department for isolation and treatment, nurses on duty needed to place the patient enthusiastically, introduce the environment and explain the necessity of isolation. Besides, nurses should evaluate the patient's pain level every 4 hours during the first 24 hours after admission, use of analgesic drugs in time, to relieve patient's pain and discomfortment, to ease the puerpera's fear and worry.

\section{Psychological Problems and Nursing Measures during the 1-2 Days after Operation}

After the cesarean section, the newborns were sent to the neonatal department for and observation. With the recovery of the puerpera spirit, worries about the newborn became an important factor causing puerpera anxiety. The transfer of a newborn to a neonatal intensive care unit was a huge mental stress for the mother. The mother did not 
know the health status of the newborn and the environment of the ward where the newborn was located. It is easy to produce negative emotions such as anxiety and depression. In response to this situation, doctor and head nurse would consult the neonatal department every day to understand the status of the newborn, and inform the mother timely. Most of the neonates were admitted to the neonatology department because of isolation observation, they showed no other complications, the with good prognosis.. In these nine cases, a baby was being broadcast live on media every day, through the live broadcast; the puerpera could keep in touch with the newborn and also benefit of relieving anxiety of the puerpera.

The Chinese have a very serious family concept. After childbirth, the mother is usually the focus of the whole family. The mother in the quarantine ward was lack of family members' companion, puerpera were prone to a strong sense of loneliness at this stage. The postpartum psychological care team members could have a deeper perception and experience of the mother's emotions and emotional states due to their past production experience. Through the three steps of inquiry, empathy, and response, empathy was used to communicate, feeling the puerpera state subjectively, alleviating the depression and anxiety effectively of women at this stage, could make the puerpera feel warm [8]. Most of the members of the postpartum psychological care team had cesarean section experience, they could predict in advance that women would face severe pain in the wound during the first 1-2 days after cesarean section .Because of the particularity of the condition; the puerpera in the quarantine ward was accompanied by no family members. In order to reduce the severe pain caused by activity after the operation, which would cause unnecessary tension and anxiety, we changed the time for removing the urinary catheter after operation from no more than 24 hours to 48 hours after the operation. We helped puerpera bedside activities immediately after removing the catheter. Before removing the urinary catheter, we encouraged and assist puerpera drinking water, guided puerpera bed and lower limb activities, in order to prevent the occurrence of deep vein thrombosis and the occurrence of urinary tract infections, to reduce postoperative discomfort and complications.

\section{Psychological Problems and Measures during the 3-7th Day after Operation}

Because of the contagious nature of Covid-19 Pneumonia, breastfeeding is not recommended after delivery during the hospitalization. Most women could understand and accepted giving up breastfeeding according to doctor's advice. But some of them felt guilty for the newborn because they could not breastfeed. We nurses used our own practical experience and professional knowledge to pacify them that the babies without breastfeeding could also grow healthily. In addition, 14-day isolation was prescribed for patients with Covid-19 pneumonia after being cured and discharged, for the health of the baby, giving artificial feeding in time was necessary. Moreover, during the process of communication, non-verbal communication such as handshake, pictures, words, etc. would be helpful for the mothers perceiving and understanding our caring.

\section{Psychological Problems and Measurements from the 8th Day after Operation to Hospital Discharging}

At this stage, the puerpera's mood would have an iterative process as the hospital stay increases. Of the 9 women, 4 women had a throat swab viral nucleic acid test that continued to be positive, and the degree of anxiety about the condition continued to increase. In the face of these patients, we have obtained the cooperation of doctors to inform the patients of the significance of the viral nucleic acid test results, the main evaluation indicators of the condition change, removed the patients' doubts in time, adjusted the treatment plan rationally, and reviewed the throat swab viral nucleic acid in time.

As neonates were gradually discharged from the neonatal department, the puerpera reduced worries about the neonate gradually, while the anxiety of some secondbirth women had not changed significantly. Maternity might makes mother worried and feel guilt for the separation from the first child [2,9]. In the process of caring for these mothers, we encouraged them, and communicated with their family in time, acquired supportment and cooperation from their family. Such as increasing the frequency of video connections between patient and family, to reduce the incidence of "isolation" of the mother. On the other hand, to relieve anxiety and depression, we prepared relevant books for the patient according to the patient's interest, suggested them watching some relaxing programs or listening light music. Drugs which improving sleeping was used to improve patient's sleeping if necessary.

\section{Psychological Problems and Nursing Measures during Fixed-Point Isolation}

According to the "New Coronavirus Pneumonia Diagnosis and Treatment Program (Trial Sixth Edition)" [10] and the requirements of Wuhan New Coronary Pneumonia Epidemic Prevention and Control Headquarters, patients need to stay at home or go to designated locations for 14 days for isolation management and health monitoring after hospital discharging. When the puerpera were isolated and managed in designated locations, we kept communication with them; we found that the anxiety of puerpera women with new 
coronary pneumonia at this stage was still more common than other patients. During the telephone follow-up, we learned that during the isolation management, these women were mainly concerned about whether the infectivity of the disease persists and whether it would affect the baby after going home. Therefore, we informed them the importance of follow-up visits after hospital discharging. Furthermore, we passed on some home protection isolation key points to them, and encouraged them to keep in touch with us if necessary to strengthen their confidence in recovery and ease their anxiety.

\section{Conclusion}

Through the careful care of the postpartum psychological care team, nine puerpera who were admitted to the quarantine ward during the hospitalization were able to alleviate the bad emotions in time. Puerpera anxiety was significantly reduced, and there was no behavior such as depression and self-injury occurred. After delivery, the mother will be isolated because of the new coronavirus pneumonia, and there will be a feeling of being isolated. We need to pay enough attention to them. The nursing experience would be helpful for nursing staff to understand the dynamic psychological variation of puerpera better, consequently promote mother-infant dyad well-being.

\section{References}

1. (2020) The National Health and Health Commission of the People's Republic of China. The National Health and Health Commission of the People's Republic of China Announcement: No. 1 in 2020 [EB / OL].

2. Hu Xiuhua, Lu Juan (2019) Social support and depression in the postpartum period of second-partured women.
Chinese Journal of Health Psychology 27(8): 1191-1194.

3. Swinson RP (2006) The GAD-7 scale was accurate for diagnosing generalised anxiety disorder. Evid Based Med 11 (6): 184.

4. Spitzer RL, Kroenke K, Williams JB, Lowe B (2006) A brief measure for assessing generalized anxiety disorder: the GAD-7 [J]. Arch Intern Med 166(10): 1092-1097.

5. Cox JL, Holden JM, Saqovsky R (1987) Detection of postnatal depression: Development of the 10-item Edinburgh Postnatal Depression Scale. Br J Psychiatry 150: 782-786.

6. Lee DT, Yip SK, Chiu HF, Leung Y, Chan KP, et al. (1998) Detecting postnatal depression in Chinese women. Validation of the Chinese version of the Edinburgh Postnatal Depression Scale. Br J Psychiatry 172: 433437.

7. Lin Min, Li Fengli, Fan Liuyi (2019) Analysis of the status quo and influencing factors of postpartum negative emotions of second-partum women [J]. Chinese Maternal and Child Health Care 34(15): 3438-3441.

8. Pan Hui, Zhou Meixiang (2017) The effect of empathic communication on anxiety and depression of uremia patients. Journal of Nursing Science 32(5): 14-16.

9. Rong W, Yanqiong $O$ (2018) A study on the quality of life of two-child elder women. Journal of Nursing, , 33 (20): 27-29.

10. (2020) Medical Administration and Hospital Authority. Notice on the issuance of a new coronavirus pneumonia diagnosis and treatment plan (for trial implementation of the sixth edition): State Health Office Medical Letter, pp: 2-19.

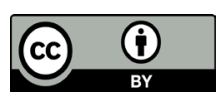

